\title{
MODELOS AGROMETEOROLÓGICOS DE ESTIMATIVA DA DURAÇÃO DO ESTÁDIO FLORAÇÃO-MATURAÇÃO PARA TRÊS CULTIVARES DE CAFÉ ARÁBICA (1)
}

\author{
FABIANA LATARO NUNES (2); MARCELO BENTO PAES DE CAMARGO $\left({ }^{\left.3,6^{*}\right)}\right.$; \\ LUIZ CARLOS FAZUOLI $\left({ }^{4,6}\right)$; GLAUCO DE SOUZA ROLIM ( $\left.{ }^{3}\right)$; \\ JOSÉ RICARDO MACEDO PEZZOPANE $\left({ }^{5}\right)$
}

\begin{abstract}
RESUMO
Modelos agrometeorológicos que relacionam condições ambientes, como temperatura do ar e disponibilidade hídrica no solo, com a fenologia do cafeeiro são importantes para subsidiar os programas de previsão de safra de café do Brasil. O objetivo foi parametrizar e testar diferentes modelos agrometeorológicos para a estimativa da duração do estádio floração-maturação para diferentes cultivares de café arábica. Dados fenológicos das cultivares Mundo Novo, Catuaí e Obatã IAC 1669-20 foram obtidos de experimentos em Campinas e Mococa nos anos agrícolas de $2001 / 2002$ a 2007/2008. Os anos de 2001/2002 a 2004/2005 foram utilizados na calibração e os de 2005/2006 a 2007/2008 no teste dos modelos. Consideraram-se três modelos de acumulação com base em evapotranspiração potencial (ETp), real (ETr), combinação entre ETr e ETp (ETr-ETp) e dois em graus-dia clássico (GD) e corrigido pelo fator hídrico (GDcorr), para considerar a influência de períodos com deficiência hídrica durante a frutificação. Resultados indicaram que os modelos com correção pelo fator hídrico (ETr, e GDcorr) tiveram estimativas da duração do estádio floração-maturação com maior consistência. Os modelos que consideram durante todo o estádio fenológico da floração-maturação valores de ETr de 746, 762 e 799 mm ou de GDcorr de 2733, 2816 e 3008 GD, respectivamente, para as cultivares Mundo Novo, Catuaí e Obatã IAC 1669-20, podem ser considerados consistentes parâmetros para a estimativa da duração deste subperíodo. Nos modelos parametrizados houve estimativas consistentes da duração do estádio floração-maturação. Os resultados confirmam a necessidade de se considerar o fator hídrico para a quantificação das somas térmicas, seja considerando ET ou graus-dia.
\end{abstract}

Palavras-chave: Coffea arabica, soma térmica, evapotranspiração, graus-dia.

\section{ABSTRACT \\ AGROMETEOROLOGICAL MODELS TO ESTIMATE THE DURATION OF FLOWERING-MATURATION STAGE FOR THREE ARABICA COFFEE CULTIVARS}

Models that consider the climatic effects to the physiologic processes of the phenology are fundamental for obtaining agrometeorological models more consistent to estimate duration of the flowering-maturation coffee growth stage and consequently better coffee grain yield estimates. The main objective was to obtain the parametrization and validation of different agrometeorological models that quantify the duration of the floweringmaturation stage for three arabica coffee cultivars. Phenological data were collected concerning Mundo Novo, Catuaí, and Obatã IAC 1669-20 cultivars from the experimental studies between the growing seasons 2001/02 to 2007/08 at Agronomic Institute (IAC/APTA), located at Campinas and Mococa, São Paulo State, Brazil. The growing seasons from 2001/02 to 2004/ 05 were used for the parametrization of the models, and from 2005/ 06 to $2007 / 08$ were reserved for the validations. Five different thermal sums were used to estimate the duration of the

(1) Recebido para publicação em 15 de maio de 2009 e aceito em 2 de março de 2010.

(2) Aluna de Mestrado no Curso de Agricultura Tropical e Subtropical, área de Concentração Tecnologia da Produção Agrícola, I AC/APTA, Caixa Postal 28, 13001-970 Campinas (SP). Com bolsa EMBRAPA/PNP\&D/CAFÉ . E-mail: fabilataro@hotmail.com

(3) Centro de Pesquisa e Desenvolvimento de Ecofisiologia e Biofísica, Instituto Agronômico, Campinas (SP). E-mail: mcamargo@iac.sp.gov.br $\left(^{*}\right)$ Autor correspondente. rolim@iac.sp.gov.br

${ }^{(4)}$ Centro de Análise e Pesquisa do Agronegócio do Café 'Alcides Carvalho', Instituto Agronômico, Campinas (SP). E-mail: fazuoli@iac.sp.gov.br

$\left(^{5}\right)$ Centro Universitário Norte do Espírito Santo, Universidade Federal do Espírito Santo, 29933-415 São Mateus (ES). Email: josepezzopane@ceunes.ufes.br

${ }^{(6)}$ Bolsista de produtividade científica CNPq. 
flowering-maturation stage. Three based on evapotranspiration: potential (ETp), actual (ETr) and a combination between ETr and ETp (ETr-ETp); and two based on classic growing degree-days (GD) and a corrected by the water availability factor (GDcorr) during the fructification period. Results indicated that models considering correction for water availability (ETr and GDcorr) presented better results with estimative of the duration of the flowering-maturation stage with larger consistence than the other sums. The accumulation models considering ETr values of $746 \mathrm{~mm}, 762 \mathrm{~mm}$, and $799 \mathrm{~mm}$ and GDcorr (base temperature of $10.5^{\circ} \mathrm{C}$ ) values of 2733 GDD, 2816 GDD, and 3008 GDD, respectively for Mundo Novo, Catuaí, and Obatã IAC $1669-20$ cultivars, can be considered more consistent parameter for flowering-maturation phase estimation. The parametrized models were tested with independent years, and presented good estimates. The results confirm the need of the water availability factor to be considered for the quantification of the required thermal sums.

Key words: Coffea arabica, thermal sum, evapotranspiration, growing degree-day.

\section{INTRODUÇÃO}

Estimativas de safras agrícolas são importantes para o estabelecimento da política cafeeira no país. Modelos agrometeorológicos que relacionam condições ambientes, como temperatura e disponibilidade hídrica no solo, com a fenologia e produtividade do cafeeiro estão sendo desenvolvidos para as regiões cafeeiras do Brasil. Esses modelos consideram que cada elemento meteorológico exerce controle na produtividade da cultura por influenciar em determinados períodos fenológicos críticos, como na floração, na formação e na maturação dos frutos dos cafeeiros.

O café arábica (Coffea arabica L.) é uma planta que leva dois anos para completar o ciclo fenológico, o qual possui uma sucessão de fases vegetativas e reprodutivas, diferentemente da maioria das plantas que emitem as inflorescências na primavera e frutificam no mesmo ano fenológico (CAMARGo, 1985). O ciclo fenológico do cafeeiro pode ser subdividido (CAMARGO e CAMARGO, 2001) para as condições climáticas tropicais do Brasil, em seis fases distintas: $1 .^{a}$ ) vegetação e formação das gemas foliares; $2^{a}{ }^{a}$ ) indução e maturação das gemas florais; $3 .^{a}$ ) florada, chumbinho e expansão dos frutos"; $4 .{ }^{\text {a) }}$ granação dos frutos; $5 .^{\text {a) }}$ maturação dos frutos e $\left.6 .^{a}\right)$ repouso e senescência dos ramos terciários e quaternários.

O período reprodutivo do cafeeiro corresponde ao subperíodo "floração-maturação (fases 3 a 5). A 3 . $^{\text {a }}$ fase - "florada, chumbinho e expansão dos frutos" - inicia-se normalmente a partir de setembro com o reinício das chuvas da primavera, que induzem a floração principal do café estendendo-se até dezembro (ZACHARIAS et al., 2008). Temperaturas do ar elevadas associadas ao déficit hídrico durante a florada podem causar abortamento das flores (CAMARGO e Pereira, 1994; Pereira et al., 2008). Após a fecundação, formam-se os chumbinhos e ocorre a expansão dos frutos. Havendo estiagem forte nesta fase, o estresse hídrico poderá prejudicar o crescimento dos frutos. A $4 .{ }^{\text {a }}$ fase - "granação dos frutos" - ocorre em pleno verão, normalmente de dezembro a março, quando a umidade interna dos frutos diminui promovendo a formação aos grãos de café. As estiagens severas nessa fase poderão resultar em má formação do endosperma (CAmargo e CAmargo, 2001). A $5 .^{\text {a }}$ fase - "maturação dos frutos" - ocorre normalmente entre abril e junho, e a maturação plena, quando pelo menos $50 \%$ dos grãos atingem a fase de cereja.

Segundo Camargo e Camargo (2001), o subperíodo "floração-maturação" é completado quando a soma de evapotranspiração potencial (ETp) atinge cerca de $700 \mathrm{~mm}$. O conceito de ETp aplicado à cultura do café pressupõe que o coeficiente cultural $(\mathrm{Kc})$ seja igual a 1, tornando a evapotranspiração da cultura (ETc) igual a ETp (AlLEN et al., 1998). Pezzopane et al. (2008) desenvolveram diferentes modelos de somas térmicas para a cultivar Mundo Novo e concluíram ser necessário considerar correção por um fator de deficiência hídrica. Porém, para ser incorporado em modelos de estimativa de produtividade são necessários mais informações para determinar com maior precisão os limites térmicos e hídricos para a maturação dos frutos de diferentes cultivares.

As cultivares Mundo Novo, Catuaí e Obatã IAC 1669-20 representam a grande maioria do parque cafeeiro do Brasil. A cultivar Mundo Novo possui alta capacidade de adaptação, com boas produções, porte alto, vigorosa, frutos vermelhos de maturação média e ótima qualidade da bebida. A cultivar Catuaí tem ampla capacidade de adaptação, com porte baixo, indicado para plantios adensados ou em renque, frutos amarelos ou vermelhos de maturação média à tardia e ótima qualidade da bebida. A cultivar Obatã IAC 1669-20 possui porte médio, boa qualidade da bebida e maturação tardia (FAZUoli et al., 2007).

Assim, este estudo teve por objetivo identificar os melhores modelos agrometeorológicos de quantificação de soma térmica na estimativa da duração do subperíodo floração-maturação dos frutos para as cultivares de café Mundo Novo, Catuaí e Obatã IAC 1669-20. 


\section{MATERIAL E MÉTODOS}

\section{Dados Fenológicos de Café}

Dados fenológicos de café das cultivares Mundo Novo, Catuaí e Obatã IAC 1669-20 foram obtidos nos arquivos do Centro de Café Alcides Carvalho e do Centro de Ecofisiologia e Biofísica de experimentos realizados no município de Campinas (Lat.: $22^{\circ} 54^{\prime} \mathrm{S}$; Long.: $47^{\circ} 05^{\prime} \mathrm{W}$ e Alt.: $674 \mathrm{~m}$ ) e em Mococa (Lat.: $21^{\circ} 28^{\prime}$ S; Long.: $47^{\circ} 01^{\prime}$ W e Alt.: $665 \mathrm{~m}$ ).

Foram utilizadas informações fenológicas de sete ciclos (anos agrícolas) como datas do início da floração plena e maturação plena, obtidas nos ciclos de $2001 / 2002$ a 2007/2008. A floração plena representava a mais importante floração de cada ano agrícola e era amostrada a data quando $50 \%$ das flores estavam abertas. A maturação plena era amostrada quando pelo menos 50\% dos grãos atingiram a fase de cereja, estando assim em condições de colheita.

Os ciclos foram divididos em duas partes, e que quatro anos agrícolas - 2001/2002 a 2004/2005- foram usados para a parametrização dos modelos em um total de seis ciclos para a cultivar Mundo Novo, sete ciclos para as cultivares Catuaí e Obatã IAC 1669-20. Desses seis ciclos da cultivar Mundo Novo, quatro foram relativos a Campinas (2001/2002 a 2004/2005) e dois ciclos relativos a Mococa (2003/2004 e 2004/2005). Já para as cultivares Catuaí e Obatã IAC 1669-20 dos sete ciclos utilizados, quatro foram relativos a Campinas (2001/2002 a 2004/2005) e três ciclos relativos a Mococa (2001/2002, 2003/2004 e 2004/ 2005).

Os ciclos relativos aos anos agrícolas de 2005/06, 2006/07e2007 / 08 foram utilizados para fazer a validação dos modelos parametrizados com dados independentes em um total de seis ciclos para as cultivares, sendo três ciclos para Campinas e três ciclos para Mococa.

\section{Somas Térmicas}

Diferentes somas térmicas foram utilizadas baseadas em evapotranspirações ("ETp", "ETr" e "ETrETp") e graus-dia ("GD" e "GDcorr corrigido pelo fator hídrico"). Inicialmente, foi determinada a acumulação da ETp, e posteriormente da ETr e acombinação dessas duas ETs (ETr-ETp) conforme proposto por PeZzopane et al. (2008) considerando ETr nos oito primeiros decêndios após a floração e o acúmulo da ETp do nono decêndio até a maturação. Este procedimento foi adotado, para considerar a influência de períodos com deficiência hídrica no desenvolvimento inicial dos frutos do cafeeiro.

Desta maneira, foram gerados valores decendiais da demanda atmosférica, representada pela ETp, estimada pelo método clássico de THORNTHWAITE (1948). A ETp é um elemento climatológico fundamental para indicar a disponibilidade de energia solar na região, constituindo assim, um índice de eficiência térmica da região, semelhante aos graus-dia (GD), porém sendo expressa em milímetros ( $\mathrm{mm}$ ) de evaporação equivalente (CAmargo e CAmargo, 2000), que é uma unidade física quantitativa .

Para a utilização dos valores acumulados de ETr e da combinação ETr-ETp, foram elaborados balanços hídricos pelo programa desenvolvido por RoLim et al. (1998), feito em planilhas no ambiente EXCEL ${ }^{\mathrm{TM}}$, com base no método de Thornthwaite e MATHER (1955), em escala decendial. Como entrada de dados, para o modelo, utilizaram-se valores diários de temperatura mínima e máxima do ar e precipitação pluvial, considerando capacidade máxima de água disponível (CAD) igual a $100 \mathrm{~mm}$, que atende à grande maioria dos solos das áreas cafeeiras do Estado de São Paulo (CAMARgo e Pereira, 1994).

Além do método de evapotranspiração utilizouse o método clássico de graus-dia (GD) que se baseia no fato de que a taxa de desenvolvimento de uma espécie vegetal está relacionada à disponibilidade de energia do meio, a qual é representada pela temperatura do ar. Para isso, pressupõe-se a existência de temperatura basal inferior, abaixo da qual a planta não se desenvolve, e se o fizer, será a taxas reduzidas (Pereira et al. 2002). Cada grau de temperatura acima da temperatura-base corresponde a um grau-dia. Cada espécie vegetal possui uma temperatura-base, que pode variar em função dos diferentes subperíodos de desenvolvimento da planta: a estimativa de GD pode ser dada pela equação:

$\mathrm{GD}=\sum \mathrm{Tmed}-\mathrm{Tb}$

em que: Tmed é a temperatura média do ar $\left({ }^{\circ} \mathrm{C}\right)$ e $\mathrm{Tb}$, a temperatura-base inferior, determinada por PEZZOPANE et al. (2008) para o período floração-maturação como de $10,2^{\circ} \mathrm{C}$. Este conceito de graus-dia assume a existência de relação linear entre desenvolvimento da cultura e temperatura, não considerando o efeito de outros fatores ambientais sobre o crescimento e desenvolvimento vegetal.

Para o cafeeiro, o desenvolvimento inicial da frutificação pode ser retardado em função de ocorrência de deficiência hídrica (Rena e Maestri, 1985). Para considerar os efeitos da ocorrência de deficiência hídrica no desenvolvimento inicial dos frutos de café foi utilizado também o "GD corrigido pelo fator hídrico" (GDcorr) que é um fator de correção para os GD, em 
função da disponibilidade de água no solo no inicio do desenvolvimento dos chumbinhos e expansão dos frutos (até o oitavo decêndio após a floração), de acordo com Pezzopane et al. (2008). O fator de correção dos graus-dia para a disponibilidade hídrica $(\mathrm{FH})$ no solo foi calculado com o uso da equação, proposta por Massignam e Angelocci (1993):

$\mathrm{FH}=\mathrm{NH} / \mathrm{N}$

em que: NH é a duração da fase que ocorreria se não houvesse deficiência hídrica e N, a duração da fase estimada por meio de equação de regressão proposta por Pezzopane et al. (2008).

Com o uso da regressão linear, na qual a duração da fase é compreendida do florescimento à maturação (N) em que $\mathrm{N}$ é a variável dependente e a razão da evapotranspiração real e a potencial (ETr/ETp), obtidas por meio do balanço hídrico sequencial decendial, nos oito primeiros decêndios, a variável independente, obteve-se uma função do tipo:

$\mathrm{N}=\mathrm{a}+\mathrm{b}(\mathrm{ETr} / \mathrm{ETp})$

Os graus-dia corrigidos (GDcorr) para os decêndios iniciais de desenvolvimento quando ocorrem deficiências hídricas, foram calculados pela equação considerando $\mathrm{Tb}$ de $10,5^{\circ} \mathrm{C}$ de acordo com Pezzopane et al. (2008):
GDcorr $=\mathrm{GD} * \mathrm{FH}$

\section{Análise Estatística}

Análises estatísticas foram realizadas considerando média, desvio-padrão (DP) e coeficiente de variação $(\mathrm{CV})$, tanto na calibração como no teste dos diferentes modelos de somas térmicas de ET e de GD.

\section{RESULTADOS E DISCUSSÃO}

\section{Parametrização dos modelos agrometeorológicos de estimativa da duração do estádio fenológico da floração-maturação}

Observando nas tabelas 1, 2 e 3, nos seis ciclos analisados para a cultivar Mundo Novo, a duração do período floração-maturação variou de 196 a 242 dias com média de 214 dias (maturação média). Considerando os sete ciclos para a cultivar Catuaí, a duração do período floração-maturação variou de 203 a 248 dias com média de 217 dias (maturação médiatardia). E dos sete ciclos analisados para a cultivar Obatã IAC 1669-20, a duração do período floraçãomaturação variou de 217 a 261 dias com média de 234 dias (maturação tardia).

Por sua vez, as necessidades térmicas, representadas por ET e GD, para as cultivares Mundo Novo, Catuaí eObatãIAC1669-20 no período da floraçãomaturação também estão apresentadas nas tabelas 1, 2 e 3. Observou-se que a acumulação média de ETp, foi de $829,4 \mathrm{~mm}$ para a cultivar Mundo Novo, de 847,7 mm para a cultivar Catuaí e $893,7 \mathrm{~mm}$ para cultivar Obatã IAC 1669-20, indicando assim que as duas últimas são

Tabela 1. Valores de acumulações de evapotranspiração (ET) e somas térmicas, em graus-dia (GD) no período floração-maturação para a cultivar Mundo Novo, em Campinas e Mococa, e análises estatísticas da parametrização dos modelos nos anos agrícolas $2001 / 2002$ a $2004 / 2005$

\begin{tabular}{|c|c|c|c|c|c|c|}
\hline \multirow{2}{*}{ Ano agrícola } & \multirow[t]{2}{*}{ Dias } & \multicolumn{3}{|c|}{ Acumulações } & \multicolumn{2}{|c|}{ Somas térmicas } \\
\hline & & ETp & Etr & Etr-Etp & GD & Gdcorr \\
\hline & n. ${ }^{\circ}$ & 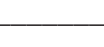 & $-\mathrm{mm}-$ & 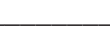 & & \\
\hline \multicolumn{7}{|l|}{ Campinas } \\
\hline $2001 / 2002$ & 206 & 775,3 & 743,6 & 755,8 & 2661,0 & 2561,3 \\
\hline $2002 / 2003$ & 196 & 817,5 & 710,5 & 730,1 & 2784,0 & 2606,7 \\
\hline $2003 / 2004$ & 207 & 770,9 & 718,4 & 739,2 & 2678,0 & 2546,3 \\
\hline $2004 / 2005$ & 211 & 812,8 & 764,2 & 796,2 & 2818,0 & 2687,3 \\
\hline \multicolumn{7}{|l|}{ Mococa } \\
\hline $2003 / 2004$ & 242 & 922,2 & 765,9 & 801,5 & 3306,0 & 3045,7 \\
\hline $2004 / 2005$ & 219 & 877,7 & 775,1 & 808,2 & 3152,0 & 2953,0 \\
\hline Média & 213,5 & 829,4 & 746,3 & 771,8 & 2899,8 & 2733,4 \\
\hline DP & 15,8 & 59,5 & 26,8 & 34,2 & 266,4 & 213,8 \\
\hline C.V. $(\%)$ & 7,4 & 7,2 & 3,6 & 4,4 & 9,2 & 7,8 \\
\hline
\end{tabular}


Tabela 2. Valores de acumulações de evapotranspiração (ET) e somas térmicas, em graus-dia (GD) no período floração-maturação para a cultivar Catuaí em Campinas e Mococa, e análises estatísticas da parametrização dos modelos nos anos agrícolas $2001 / 2002$ a $2004 / 2005$

\begin{tabular}{|c|c|c|c|c|c|c|}
\hline \multirow{2}{*}{ Ano Agrícola } & \multirow[t]{2}{*}{ dias } & \multicolumn{3}{|c|}{ Acumulações } & \multicolumn{2}{|c|}{ Somas térmicas } \\
\hline & & ETp & ETr & ETr-ETp & GD & GDcorr \\
\hline & n. ${ }^{\circ}$ & 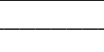 & $-\mathrm{mm}-$ & 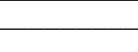 & & \\
\hline \multicolumn{7}{|l|}{ Campinas } \\
\hline $2001 / 2002$ & 213 & 805,4 & 773,4 & 785,9 & 2802,0 & 2699,3 \\
\hline $2002 / 2003$ & 203 & 848,0 & 724,4 & 745,4 & 2914,0 & 2712,0 \\
\hline $2003 / 2004$ & 206 & 850,1 & 757,6 & 790,1 & 3038,0 & 2840,3 \\
\hline $2004 / 2005$ & 204 & 788,1 & 746,5 & 771,5 & 2709,0 & 2592,3 \\
\hline \multicolumn{7}{|l|}{ Mococa } \\
\hline $2001 / 2002$ & 220 & 837,8 & 766,1 & 832,6 & 2971,0 & 2810,8 \\
\hline $2003 / 2004$ & 248 & 941,6 & 785,3 & 820,9 & 3390,0 & 3126,7 \\
\hline $2004 / 2005$ & 226 & 863,2 & 778,6 & 829,1 & 3109,0 & 2927,0 \\
\hline Média & 217,1 & 847,7 & 761,7 & 796,5 & 2990,4 & 2815,5 \\
\hline DP & 16,0 & 49,1 & 20,9 & 32,5 & 222,4 & 175,1 \\
\hline C.V. $(\%)$ & 7,4 & 5,8 & 2,8 & 4,1 & 7,4 & 6,2 \\
\hline
\end{tabular}

Tabela 3. Valores de acumulações de evapotranspiração (ET) e somas térmicas, em graus-dia (GD) no período floração-maturação para a cultivar Obatã IAC 1669-20 em Campinas e Mococa, e análises estatísticas da parametrização dos modelos nos anos agrícolas $2001 / 2002$ a $2004 / 2005$

\begin{tabular}{|c|c|c|c|c|c|c|}
\hline \multirow{2}{*}{ Ano Agrícola } & \multirow[t]{2}{*}{ dias } & \multicolumn{3}{|c|}{ Acumulações } & \multicolumn{2}{|c|}{ Somas térmicas } \\
\hline & & ETp & $\mathrm{ETr}$ & ETr-ETp & GD & GDcorr \\
\hline & $\mathrm{n} .^{\circ}$ & 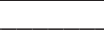 & $-\mathrm{mm}-$ & 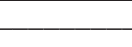 & & \\
\hline \multicolumn{7}{|l|}{ Campinas } \\
\hline $2001 / 2002$ & 242 & 891,6 & 839,5 & 872,1 & 3224,0 & 3081,0 \\
\hline $2002 / 2003$ & 231 & 930,4 & 797,2 & 827,8 & 3294,0 & 3068,4 \\
\hline $2003 / 2004$ & 217 & 887,7 & 793,8 & 827,7 & 3192,0 & 2985,3 \\
\hline $2004 / 2005$ & 225 & 835,6 & 775,5 & 819,0 & 2922,0 & 2771,0 \\
\hline \multicolumn{7}{|l|}{ Mococa } \\
\hline $2001 / 2002$ & 227 & 861,6 & 777,6 & 856,4 & 3098,0 & 2913,7 \\
\hline $2003 / 2004$ & 261 & 963,7 & 807,4 & 843,0 & 3481,0 & 3214,7 \\
\hline $2004 / 2005$ & 238 & 885,0 & 800,4 & 850,9 & 3207,0 & 3022,0 \\
\hline Média & 234,4 & 893,7 & 798,8 & 842,4 & 3202,6 & 3008,0 \\
\hline DP & 14,4 & 42,4 & 21,4 & 18,8 & 171,3 & 139,9 \\
\hline C.V. $(\%)$ & 6,1 & 4,7 & 2,7 & 2,2 & 5,4 & 4,6 \\
\hline
\end{tabular}

mais tardias que a cultivar Mundo Novo. Estes valores foram superiores ao valor originalmente sugerido por Camargo e Camargo (2001) de cerca de $700 \mathrm{~mm}$.

As acumulações de ETp representaram valores de DP e de CV muito superiores comparados com as acumulações utilizando ETr ou ETr-ETp para as três cultivares consideradas, indicando assim desempenho inferior da soma térmica de ETp. Por outro lado, comparando as acumulações de ETr e ETr-ETp, ETr se destaca com melhor desempenho. Assim, os acúmulos considerando valores médios de ETr de $746,3 \mathrm{~mm}$, $761,7 \mathrm{~mm}$ e $798,8 \mathrm{~mm}$ para as cultivares Mundo Novo,
Catuaí e Obatã IAC 1669-20, respectivamente, podem ser considerados melhores indicativos para a estimativa do período floração-maturação do que pelo acúmulo de ETp ou de ETr-ETp.

Com relação ao método clássico de GD utilizando $\mathrm{Tb}$ de $10,2{ }^{\circ} \mathrm{C}$, observaram-se médias de $2.899,8 \mathrm{GD}$ para a cultivar Mundo Novo, 2.990,4 GD para a cultivar Catuaí e 3.202,6 GD para cultivar Obatã IAC 166920. A parametrização utilizando somas térmicas com procedimento de correção $\left(\mathrm{Tb}=10,5^{\circ} \mathrm{C}\right)$ para o fator hídrico proporcionou melhor desempenho, com valores inferiores de DP e CV. Para a cultivar Mundo Novo as 
análises estatísticas resultaram valores de DP de 213,8 $\mathrm{mm}, \mathrm{CV}$ de 7,8\% e valor médio de soma de GDcorr de 2733,4 GD. Resultados semelhantes foram obtidos por Pezzopane et al. (2008) que determinaram o valor médio de GDcorr de 2761,0 GD.

Assim, as somas térmicas baseadas nos valores médios de GDcorr $\left(\mathrm{Tb}=10,5^{\circ} \mathrm{C}\right)$ considerando procedimento de correção para o fator hídrico de 2733,4 GD, 2815,5 GD e 3008,0 GD para as cultivares Mundo Novo, Catuaí e Obatã IAC 1669-20, respectivamente, podem ser considerados melhores indicativos para a estimativa do período floração-maturação do que a soma térmica considerando o método clássico de GD.

\section{Testes dos modelos agrometeorológicos de estimativa da duração do estádio fenológico da floração- maturação}

Os modelos parametrizados foram testados considerando dados de anos independentes de $2005 / 2006$ a 2007/2008. Os valores acumulados de ETp avaliados neste período foram de $820 \mathrm{~mm}, 840 \mathrm{~mm}$ e $856 \mathrm{~mm}$ para as cultivares Mundo Novo, Catuaí e Obatã IAC 1669-20 respectivamente.
Nas figuras 1, 2 e 3 observam-se os resultados dos desvios dos modelos em relação aos dados observados (2005/2006 a 2007/2008) para as cultivares Mundo Novo, Catuaí e Obatã IAC 1669-20 para Campinas e Mococa. Observou-se que os modelos com os menores desvios nos diferentes anos testados foram ETr, ETrETp e GDcorr.

No ano agrícola de 2007/2008 observaram-se os maiores desvios nas acumulações de ETp (150 a $180 \mathrm{~mm}$ ) e GD (380 a 500 GD), especialmente para Campinas, devido às deficiências hídricas que ocorreram em outubro e novembro de 2007, as quais interferiram na duração do estádio fenológico da floração-maturação.

As análises estatísticas dos testes indicaram que as somas de ETp e de GD resultaram também em valores de DP e CV muito superiores comparados com os demais modelos, semelhantes aos anotados por Pezzopane et al. (2008) para a cultivar Mundo Novo.

Estes resultados confirmam a necessidade de se considerar o fator hídrico para a quantificação das acumulações e somas térmicas necessárias para estimação do período floração-maturação do café.
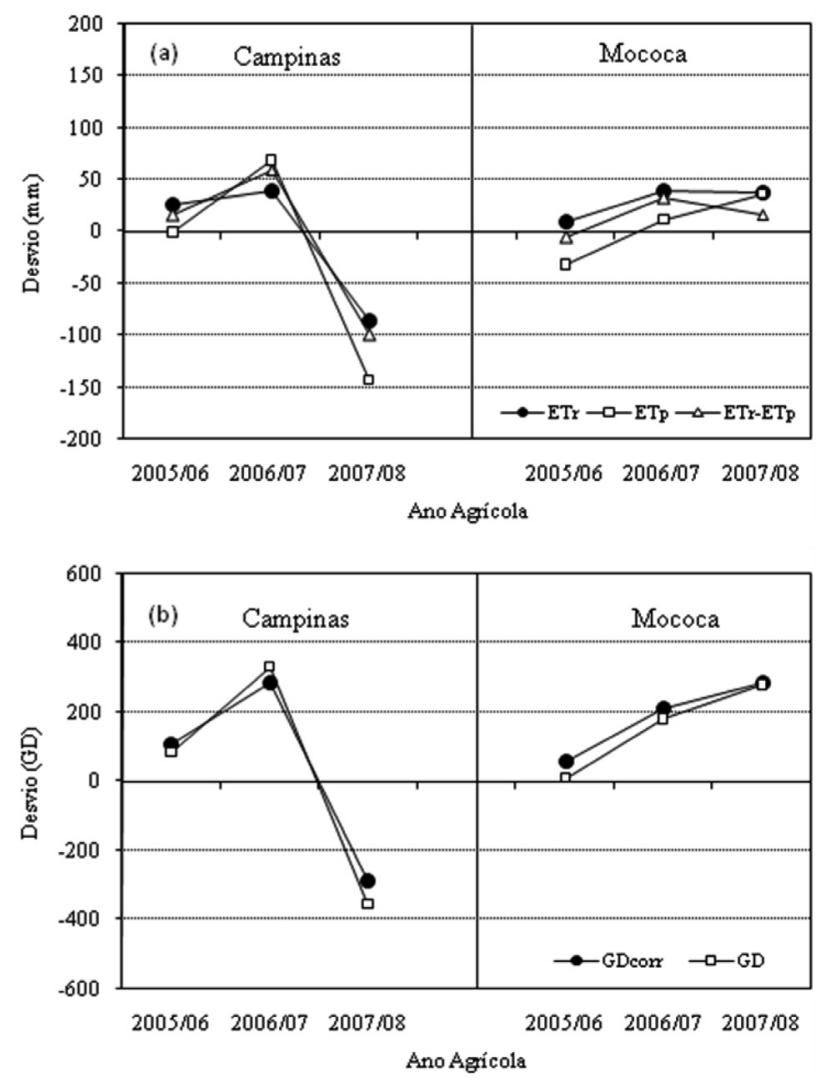

Figura 1. Valores dos desvios em relação à média obtidos pelas análises estatísticas para a cultivar Mundo Novo com base nas acumulações de ETp, ETr, ETr-ETp (a) e nas somas térmicas GD e GDcorr (b) para Campinas e Mococa, no período de 2005 / 2006 a $2007 / 2008$, como teste do modelo parametrizado. 

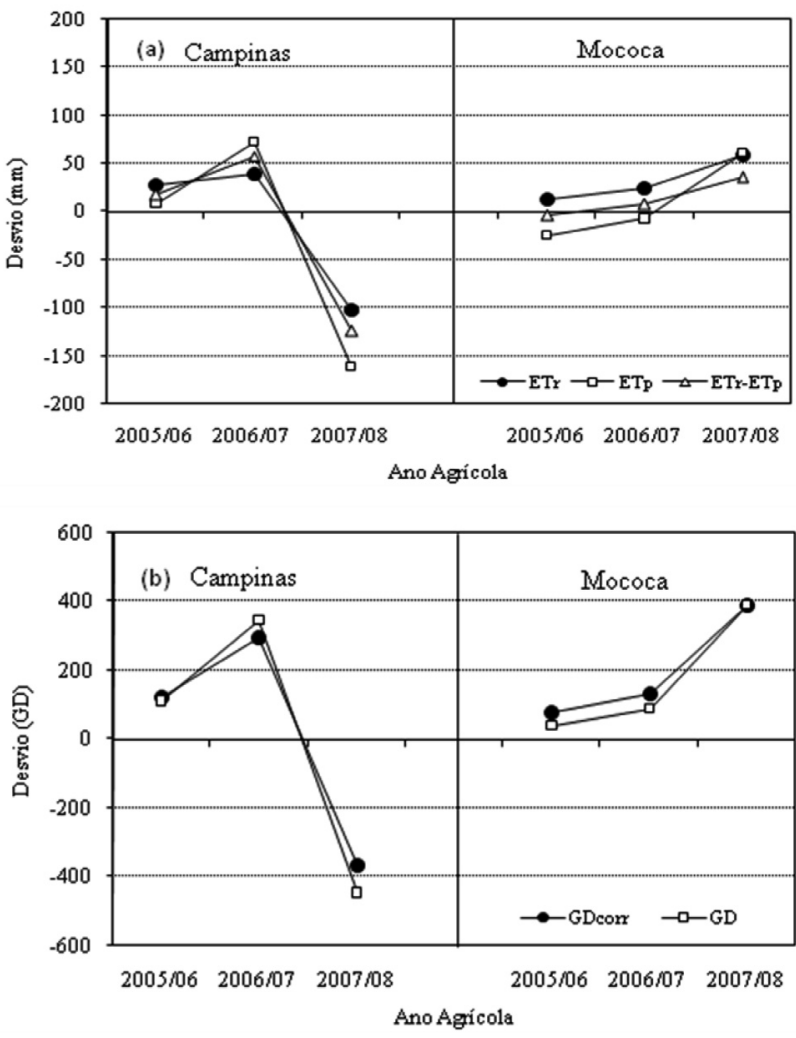

Figura 2. Valores dos desvios em relação à média obtidos pelas análises estatísticas para a cultivar Catuaí com base nas acumulações de ETp, ETr, ETr-ETp (a) e nas somas térmicas GD e GDcorr (b) para Campinas e Mococa, no período de 2005/ 2006 a 2007 / 2008, como teste do modelo parametrizado.

\section{CONCLUSÕES}

1. Os modelos de soma térmica considerando correções pelo fator hídrico (ETr, ETr-ETp e GDcorr) estimam com mais consistência a duração do ciclo da floração-maturação do café do que os demais (ETp e GD), confirmando a necessidade de se considerar o fator hídrico para a quantificação das somas térmicas.

2. O modelo de soma térmica, considerando ETr durante todo o ciclo da floração-maturação, proporciona para as três cultivares melhor desempenho comparado com os demais modelos.

3. As acumulações térmicas necessárias para o complemento do estádio floração-maturação dos frutos são $746 \mathrm{~mm}$ (ETr) ou 2733 GD (GDcorr) para a cultivar Mundo Novo; $762 \mathrm{~mm}$ (ETr) ou 2816 GD (GDcorr) para a cultivar Catuaí; e 799 mm (ETr) ou 3008 GD (GDcorr) para a cultivar Obatã IAC 1669-20.
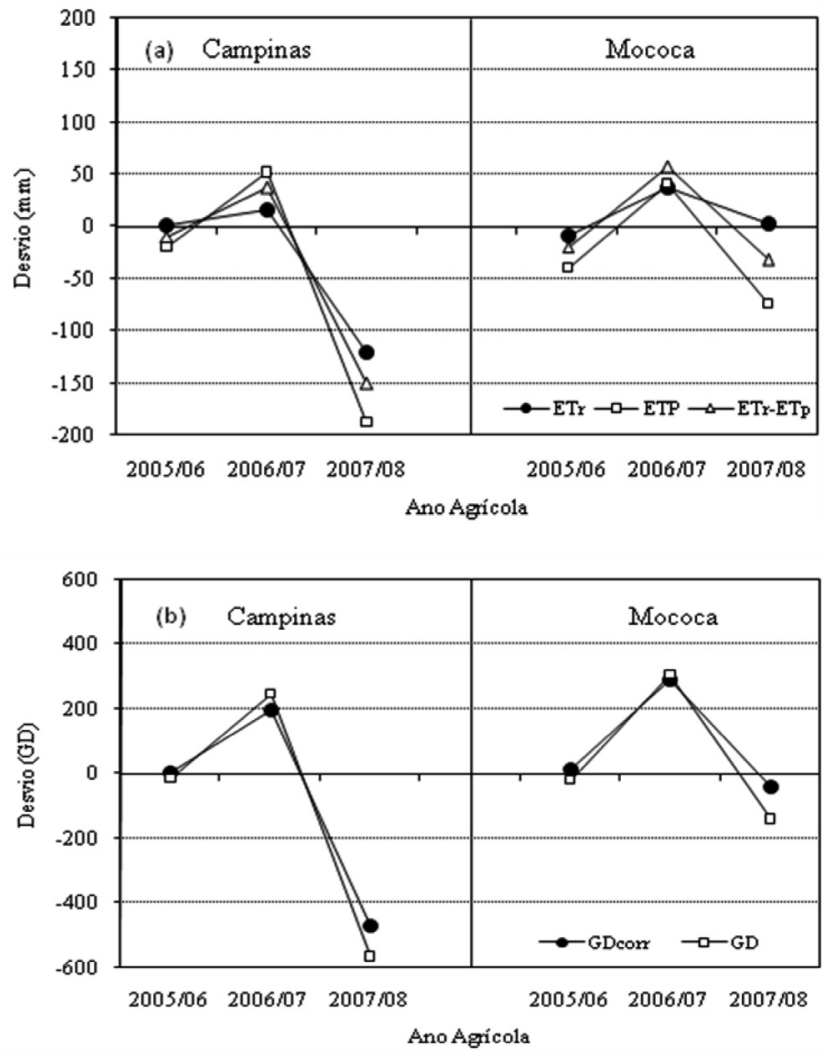

Figura 3. Valores dos desvios em relação à média obtidos pelas análises estatísticas para a cultivar Obatã IAC 166920 com base nas acumulações de ETp, ETr, ETr-ETp (a) e nas somas térmicas GD e GDcorr (b) para Campinas e Mococa, no período de 2005/ 2006 a 2007 / 2008, como teste do modelo parametrizado.

\section{AGRADECIMENTOS}

Ao Consórcio Brasileiro de Pesquisa e Desenvolvimento do Café (EMBRAPA/CBP\&D / CAFÉ) pelo financiamento do projeto.

\section{REFERÊNCIAS}

ALLEN, R.G.; PEREIRA, L.S.; RAES, D.; SMITH, M. Crop evapotranspiration: Guidelines for predicting crop water requirements. Rome: FAO, 1998. 300p. (Irrigation and Drainage Paper, 56)

CAMARGO, A.P. Florescimento e frutificação do café arábica nas diferentes regiões cafeeiras do Brasil. Pesquisa Agropecuária Brasileira, v.20, p.831-839, 1985.

CAMARGO, A.P.; CAMARGO, M.B.P. Uma revisão analítica da evapotranspiração potencial. Artigo de Revisão. Bragantia, v.59, p.125-137, 2000. 
CAMARGO, A.P.; CAMARGO, M.B.P. Definição e esquematização das fases fenológicas do cafeeiro arábica nas condições tropicais do Brasil. Bragantia, v.60, p.65-68, 2001.

CAMARGO, A.P.; PEREIRA, A.R. Agrometeorology of the coffe crop. Geneve: World Meteorological Organization, 1994. 96p. (Agricultural Meteorology CaM report, 58)

FAZUOLI, L.C.; SILVAROLA, M.B., SALVA, T.J.G.; GUERREIROFILHO,O.;MEDINAFILHO,H.P.;GONÇALVES, W. Cultivares de café arábica do IAC: Um patrimônio da cafeicultura brasileira. O Agronômico, v.59, p. 12-15, 2007.

MASSIGNAM, A.M.; ANGELOCCI, L.R. Relações entre temperatura do ar, disponibilidade hídrica no solo, fotoperíodo e duração de subperíodos fenológicos do girassol. Revista Brasileira de Agrometeorologia, v.1, p.63-69, 1993.

PEZZOPANE, J.R.M.; PEDRO JUNIOR, M.J.; CAMARGO, M.B.P.; FAZUOLI, L.C. Exigência térmica do café Arábica cultivar Mundo Novo no subperíodo florescimento-colheita. Ciência e Agrotecnologia, v.32, p.1781-1786, 2008.

PEREIRA, A.R.; ANGELOCCI, L.R.; SENTELHAS, P.C. Agrometeorologia: Fundamentos e Aplicações Práticas. Guaíba: Agropecuárias. 2002. 465p.
PEREIRA, A. R.; CAMARGO, A.P.; CAMARGO, M.B.P. Agrometeorologia de Cafezais no Brasil. Campinas: Instituto Agronômico. 2008. 127p.

RENA, A.B.; MAESTRI, M. Fisiologia do cafeeiro. Informe Agropecuário, v.11, p.26-40, 1985.

ROLIM, G.S.; SENTELHAS, P.C.; BARBIERI, V. Planilhas no ambiente EXCEL ${ }^{\mathrm{TM}}$ para os cálculos de balanços hídricos: normal, seqüencial, de cultura e de produtividade real e potencial. Revista Brasileira de Agrometeorologia, v.6, p.133137, 1998

THORNTHWAITE, C.W. An approach toward a rational classification of climate. Geography Review, v.38, p.55-94, 1948.

THORNTHWAITE, C.W.; MATHER, J.R. The water balance. Centerton, NJ: Drexel Institute of Technology - Laboratory of Climatology, 1955. 104p. (Publications in Climatology, v. 8, n.1)

ZACHARIAS, A.O.; CAMARGO, M.B.P.; FAZUOLI, L.C. Modelo agrometeorológico de estimativa do início da florada plena do cafeeiro. Bragantia, v.67, p.249-256, 2008. 